\title{
SUMMARY OF THE MANITOBA 1971 CHRISTMAS BIRD COUNT
}

Christmas Bird Counts (December 18, 1971, to January 2, 1972) were made in 25 localities in Manitoba. In four localities, Winnipeg, Minnedosa, Pinawa and Riding Mountain two or more field parties had been organized. The counts were compiled and submitted to the Blue Jay but they were not received in time for the March issue. This report lists the localities in which counts were made and the bird species recorded. The numbers following the species names give the number of individuals seen and in brackets the number of localities in which they were recorded. If you wish more detail on the 1971 Manitoba Christmas Bird Count or if you have suggestions re publication of the counts, write to H. W. R. Copeland, Manitoba Museum of Man and Nature, 190 Rupert Avenue, Winnipeg, Manitoba, R3B 0N2.

Localities in Manitoba in which 1971 Christmas Bird Counts were taken include the following 25 areas: Balmoral, Brandon, Deleau, Delta, Douglas, Glenora, Gypsumville, Hartney, Kenville, Laurier, LockportSelkirk-Birdshill Park, Lorette, Lyleton, Miami, Minnedosa, Oak Lake, Pinawa, Reston, Riding Mountain National Park, Roland, St. Norbert, Sandy Hook, Souris, Stonewall and Winnipeg.

Birds seen during the 1971 Manitoba Christmas Bird Count include the following 65 species. (Numbers in brackets refer to the number of localities in which the species was recorded) : Canada Goose 16(2); Mallard 19(2); Goshawk 5(4); Redtailed Hawk 2(2); Rough-legged Hawk 1(1); Golden Eagle 3(2); Marsh Hawk 1(1); Gyrfalcon 1(1); Pigeon Hawk 3(3); Spruce Grouse $2(2)$; Ruffed Grouse 55(12) ; Sharptailed Grouse 267(11); Ring-necked Pheasant 13(3); Gray Partridge 102(9); Turkey 24(2); Rock Dove $247(4)$; Mourning D o v e 8(3); Screech Owl 1(1); Great Horned
Owl 21(11); Snowy Owl 24(10); Great Gray Owl 1(1); Short-eared O w l 2(1); Pileated Woodpecker 11(5); Hairy Woodpecker 92(23); Downy Woodpecker 83(21); Blackbacked Three-toed Woodpecker 2(1); Northern Three-toed Woodpecker 1(1) ; Horned Lark 12(3) ; Gray Jay $52(3)$; Blue Jay 377(19); Blackbilled Magpie. 353(21); Common Raven 263(9); Common Crow 107(4); Black - capped Chickadee 796(25); Boreal Chickadee 39(2); White-breasted Nuthatch $80(15)$; Red-breasted Nuthatch 21(3); Brown Creeper 5(2); Brown Thrasher 1(1); American Robin 2(2); Varied Thrush 1(1); Golden-crowned Kinglet 3(2); Bohemian Waxwing 256(4); Cedar Waxwing 4(1); Northern Shrike 7(7); Starling 362(11); House Sparrow 5,804(21); Western Meadowlark 5 (5); Yellow-headed Blackbird 3(1) ; Red-winged Blackbird 1(1); Rusty Blackbird 3(1); Common Grackle $3(2)$; Brown-headed Cowbird 1(1); Rose-breasted Grosbeak 1(1); Evening Grosbeak 1,030(17); Pine Grosbeak 403(20); Hoary Redpoll 39(7); Common Redpoll 2,341(19); Pine Siskin 2(1); American Goldfinch 4(1); White - winged Cross bill 177(10); Slate-colored Junco 2(2); Harris' Sparrow 1(1); Song Sparrow $1(1)$; Snow Bunting 1,956(14).

\section{REQUEST FOR INFORMATION RE SHOREBIRDS RECAPTURE OPERATION}

Several species of shorebirds will be caught on the Magdalen Islands (Gulf of St. Lawrence) and marked with yellow feather dye or yellow leg streamers or both. Reports of sight records of these marked birds would be appreciated. Please give locality, date, species, name and address of observer(s) and if the bird is captured, weight and band number.

Information should be sent to: Raymond McNeil, Centre de Recherches Ecologiques de Montréal, 4101 est, rue Sherbrooke, Montréal 406, Que., Canada. 